Supporting Information

\title{
CoFe Nanparticle-decorated reduced Graphene Oxide for the highly efficient reduction of 4-Nitrophenol
}

Chuncai Konga, Weijie Lei ${ }^{\mathrm{a}}$, Bosen Lei ${ }^{\mathrm{a}}$, Fangzhao Pu${ }^{\mathrm{a}}$, Ge Wang ${ }^{\mathrm{b}}$, Xiaojing Zhang ${ }^{\mathrm{a}}$,

Cheng Zhou ${ }^{\mathrm{b}, *}$ and Zhimao Yang ${ }^{\mathrm{a}, *}$

${ }^{a}$ Ministry of Education Key Laboratory for Non-equilibrium Synthesis and Modulation

of Condensed Matter, Shaanxi Province Key Laboratory of Advanced Functional

Materials and Mesoscopic Physics, School of Physics, Xi'an Jiaotong University, Xi'an

710049, China.

${ }^{b}$ Beijing Institute of Control Engineering, Beijing 100190, China.

*Corresponding Author

E-mail: zmyang@xjtu.edu.cn (Z.M. Yang), zhoucheng0208@163.com (C. Zhou) 


\section{Table of contents}

1. Figure S1. Low-magnification SEM images (a) CoFe/RGO-200, (b) CoFe/RGO400 and (c) CoFe/RGO-600.

2. Figure S2. Size distributions of (a) CoFe/RGO-400 and (b) CoFe/RGO-600.

3. Table S1 Magnetic properties

4. Figure S3. TG curves of $\mathrm{Co}, \mathrm{Fe}$ and RGO precursor.

5. Table $\mathbf{S 2}$ Chemical analysis of $\mathrm{CoFe} / \mathrm{RGO}$ by ICP-MS

6. Figure S4. Time-dependent UV-vis absorption spectra of 4-NP reduction to 4-AP in the presence of $\mathrm{CoFe} / \mathrm{RGO}-400$ from the 1 st to the $6^{\text {th }}$ reaction cycle.

7. Figure S5. Time-dependent UV-vis absorption spectra of 4-NP reduction to 4-AP in the presence of $\mathrm{CoFe} / \mathrm{RGO}-600$ from 1 st to the $6^{\text {th }}$ reaction cycle.

8. Figure S6. HPLC chromatogram of 4-NP and its degradation products at a predetermined reaction time.

9. Figure S7. Reaction rates based on the real $\mathrm{CoFe}$ content in the samples. 

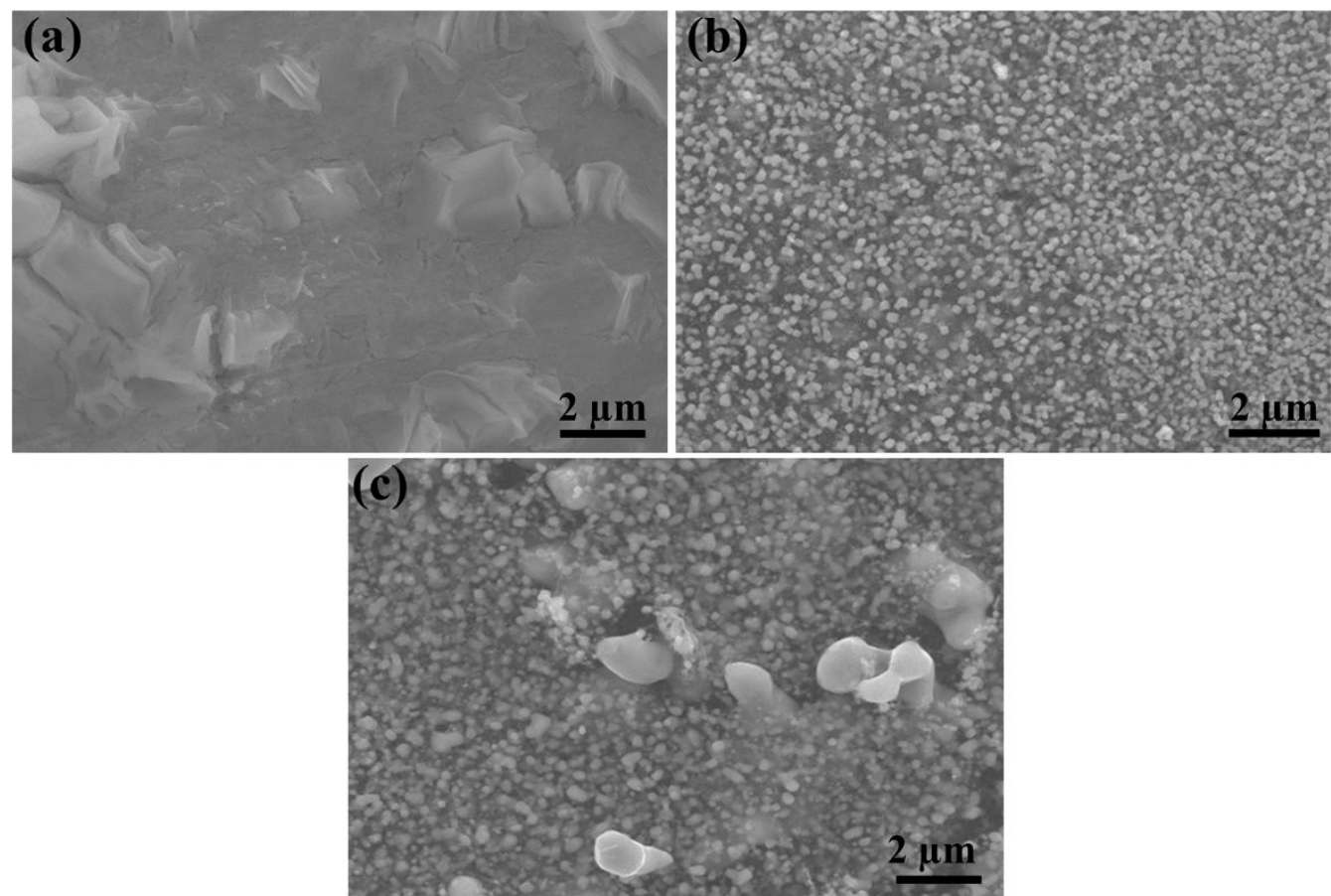

Figure S1. Low-magnification SEM images (a) CoFe/RGO-200, (b) CoFe/RGO-400 and (c) CoFe/RGO-600.
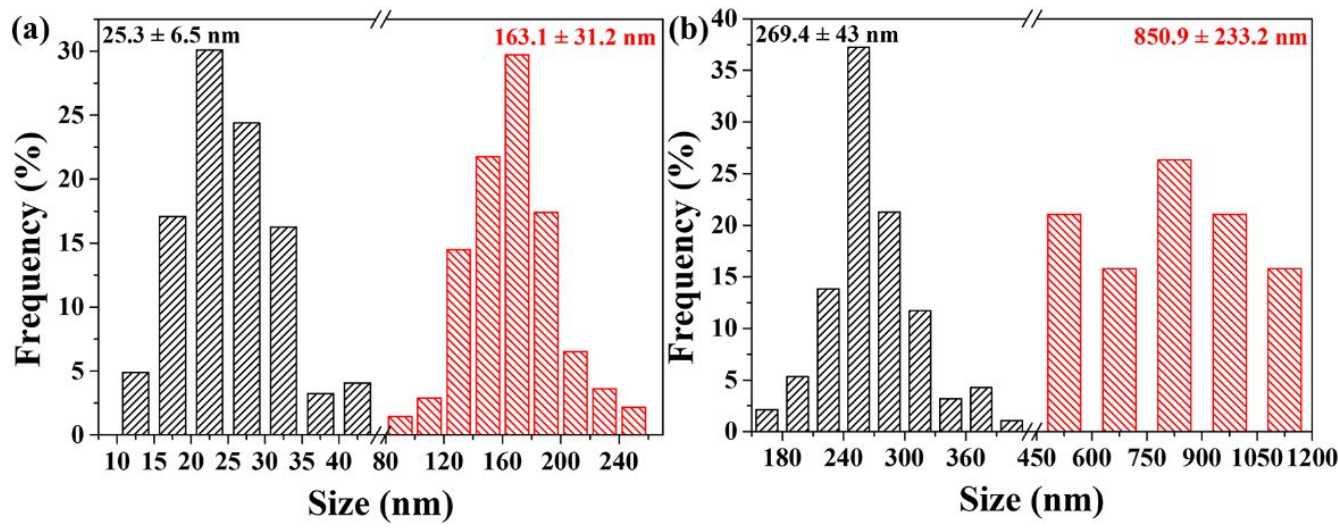

Figure S2. Size distributions of (a) CoFe/RGO-400 and (b) CoFe/RGO-600. 
Table S1 Magnetic properties

\begin{tabular}{ccccc}
\hline Sample & Ms (emu g-1 & Mr $\left(\mathrm{emu} \mathrm{g}^{-1}\right)$ & $\mathrm{Mr} / \mathrm{Ms}$ & $\mathrm{Hc}(\mathrm{Oe})$ \\
\hline CoFe/RGO-200 & 1.13 & 0.05 & 0.04 & 804.8 \\
$\mathrm{CoFe} / \mathrm{RGO}-400$ & 178.8 & 37.4 & 0.21 & 823.2 \\
$\mathrm{CoFe} / \mathrm{RGO}-600$ & 204.7 & 37.5 & 0.18 & 824.3 \\
\hline
\end{tabular}

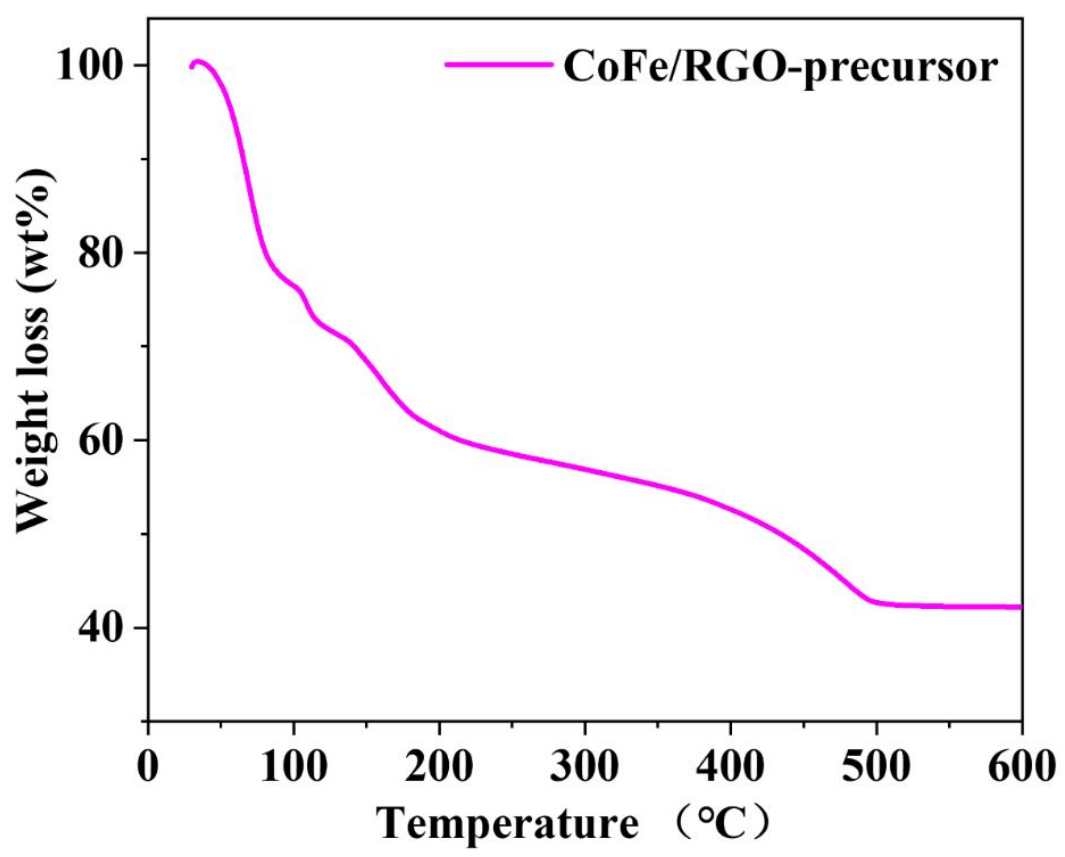

Figure S3. TG curves of $\mathrm{Co}, \mathrm{Fe}$ and RGO precursor.

Table S2 Chemical analysis of CoFe/RGO by ICP-MS

\begin{tabular}{ccccc}
\hline Sample & Co $(w t \%)$ & Fe $(w t \%)$ & CoFe (wt\%) & rGo (wt\%) \\
\hline CoFe/RGO-200 & 14.15 & 15.00 & 29.15 & 70.85 \\
CoFe/RGO-400 & 29.30 & 36.47 & 65.77 & 34.23 \\
CoFe/RGO-600 & 30.13 & 37.70 & 67.83 & 32.17 \\
\hline
\end{tabular}



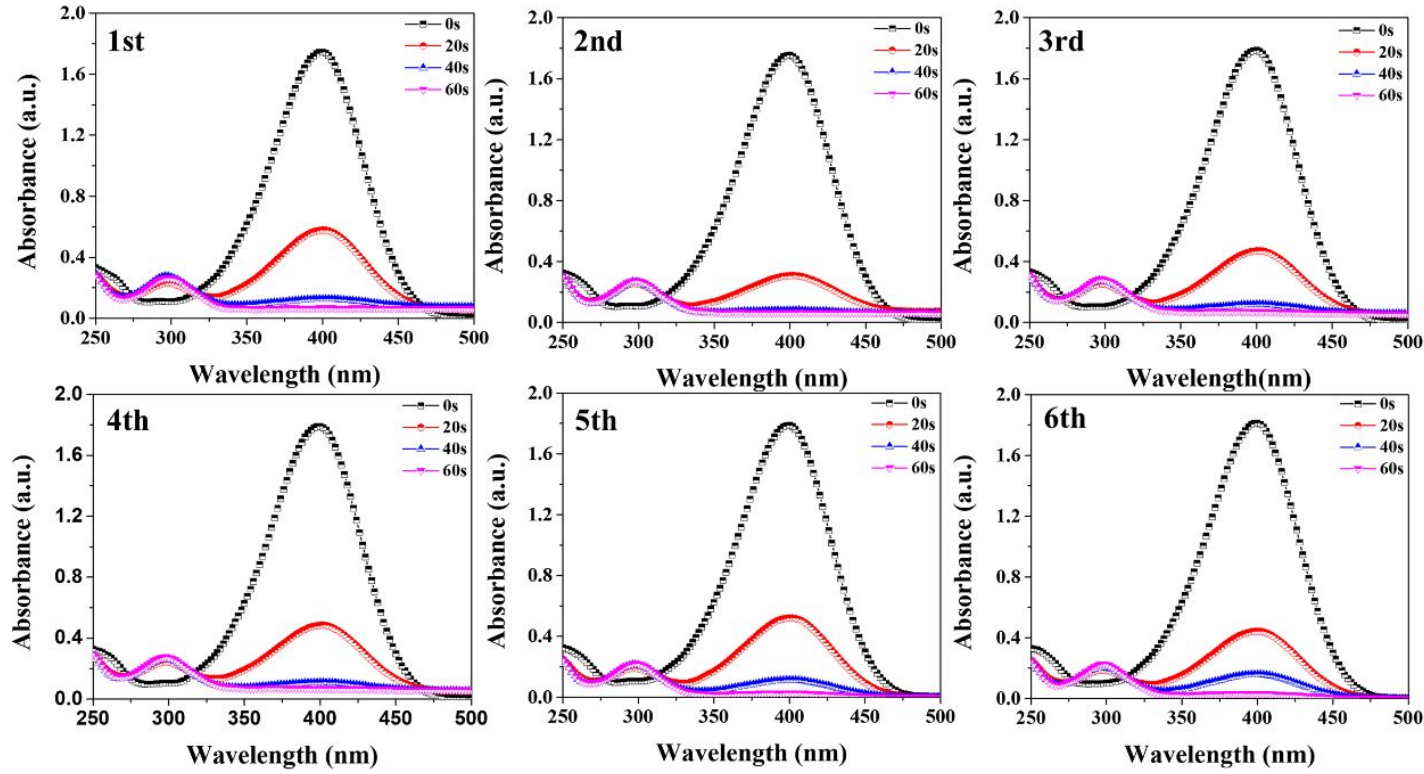

Figure S4. Time-dependent UV-vis absorption spectra of 4-NP reduction to 4-AP in the presence of $\mathrm{CoFe} / \mathrm{RGO}-400$ from the 1 st to the $6^{\text {th }}$ reaction cycle.
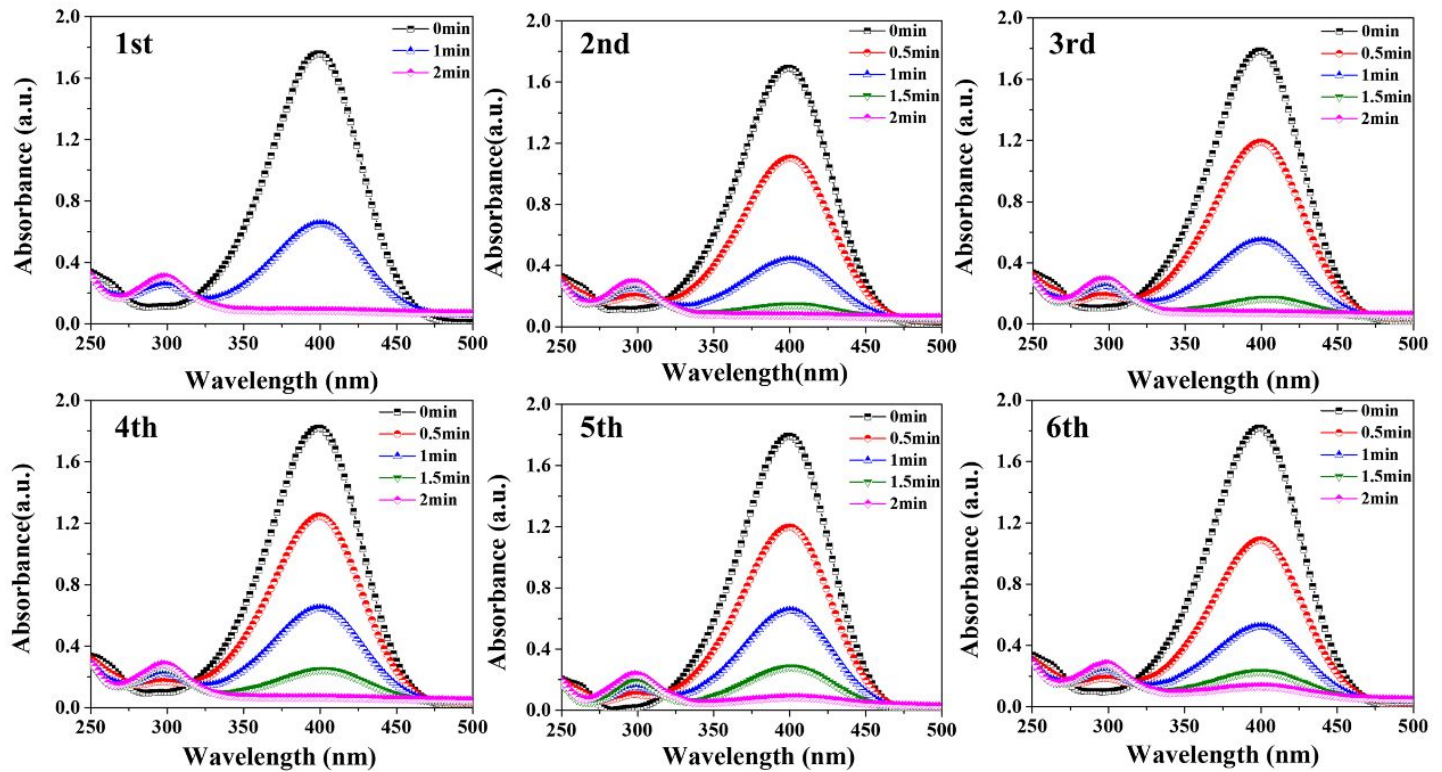

Figure S5. Time-dependent UV-vis absorption spectra of 4-NP reduction to 4-AP in the presence of $\mathrm{CoFe} / \mathrm{RGO}-600$ from 1 st to the $6^{\text {th }}$ reaction cycle. 


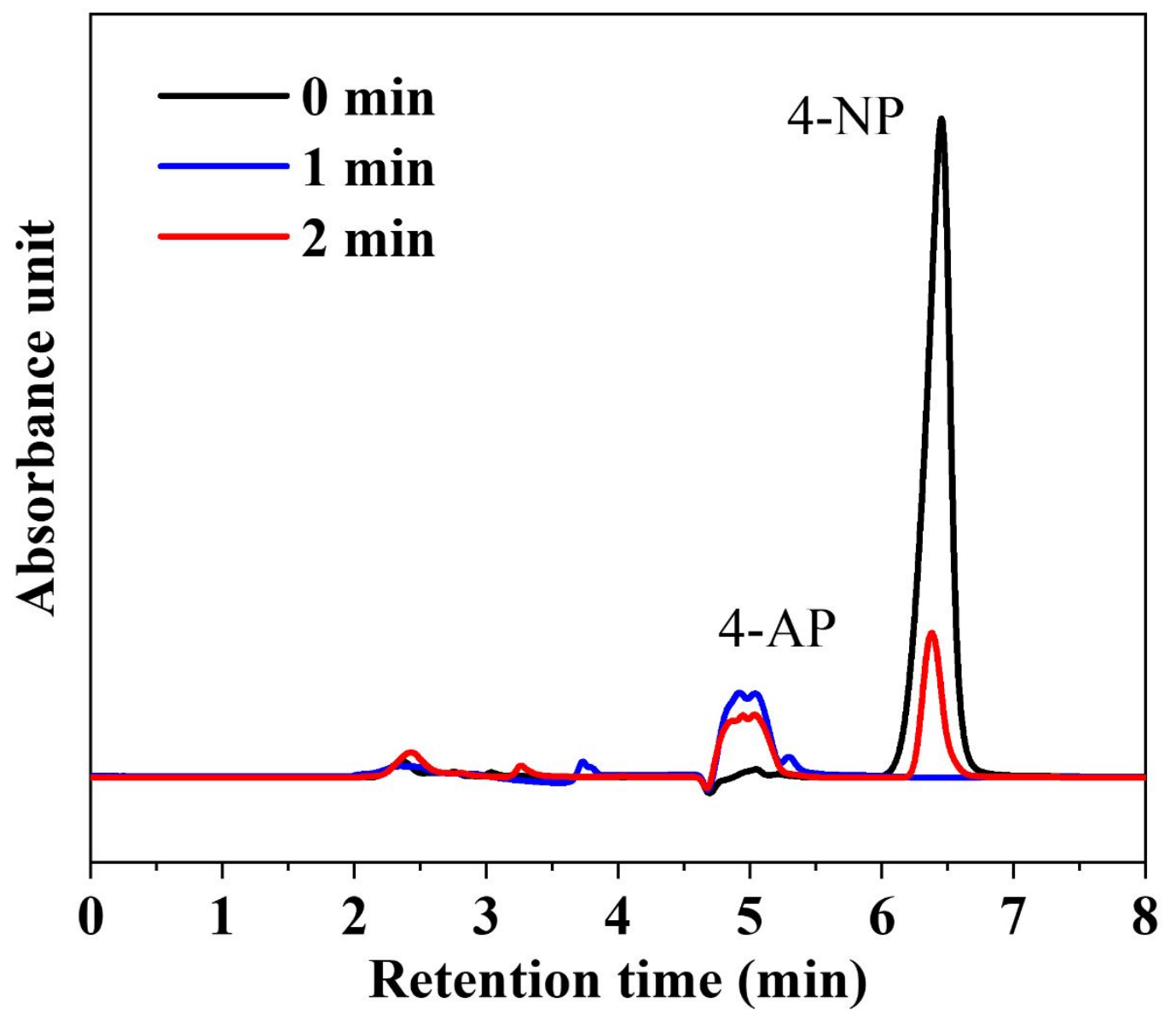

Figure S6. HPLC chromatogram of 4-NP and its degradation products at a predetermined reaction time. 


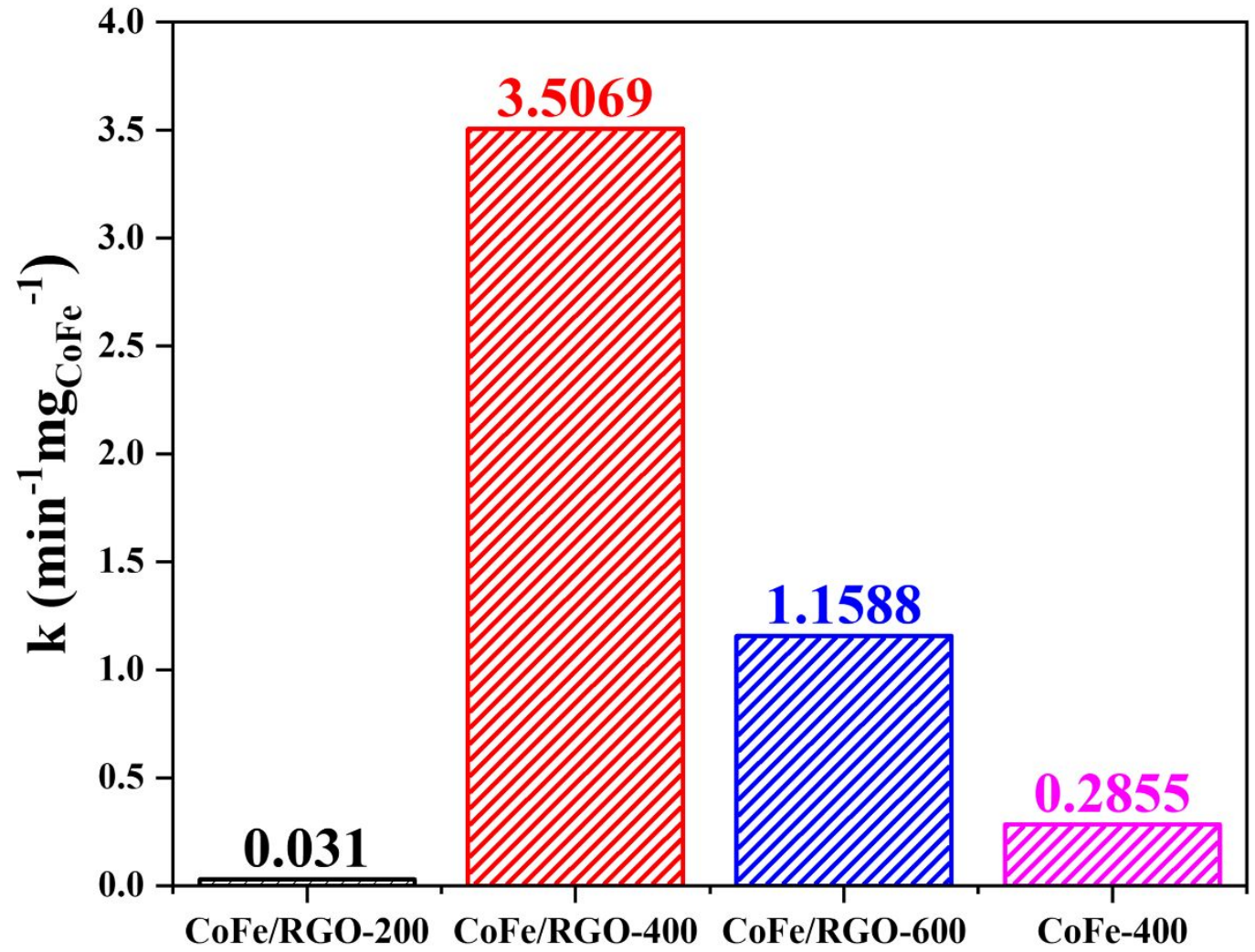

Figure S7. Reaction rates based on the real CoFe content in the samples. 